\title{
Filariose bancroftiana em áreas urbanas do Estado de Alagoas, nordeste do Brasil: estudo em população geral
}

\author{
Bancroftian filaniasis in urban areas of Alagoas State, \\ Northeast Brazil: study in general population
}

\author{
Eliana M. Mauricio da Rocha', Gilberto Fontes', Ana C. Brito', \\ Tatiana R.C. Silva', Zulma Medeiros ${ }^{2}$ e Carlos M.F. Antunes ${ }^{3}$
}

\begin{abstract}
Resumo Com objetivo de estudar a situação da filariose linfática em Alagoas, foi realizado um inquérito hemoscópico na população geral de áreas urbanas das três diferentes regiões fisiográficas do estado. Dos 101 municípios, foram pesquisados aleatoriamente 10, sendo a bancroftose detectada somente na capital, Maceió. Em um estudo seccional feito com a população geral de 4 bairros desta cidade foram examinados 10.973 indivíduos sendo detectados 226 microfilarêmicos, com prevalências nos bairros variando de 0 a 5,4\%. Tanto a prevalência de microfilarêmicos como a microfilaremia média foram significativamente maiores em indivíduos do sexo masculino. Entre os examinados não nascidos em Maceió, o tempo de residência na área endêmica foi significativamente maior entre microfilarêmicos que entre amicrofilarêmicos. Baseado nestes dados, medidas de controle já foram implementadas visando a eliminação da filariose linfática na região.
\end{abstract}

Palavras-chaves: Wuchereria bancrofti. Filariose linfática. Bancroftose. Epidemiologia.

Abstract In order to assess the present status of lymphatic filariasis in Alagoas State, Brazil, hemoscopic surveys were carried out in the human population of the three different physiographic regions of the State. Blood samples were collected by thick smear technique taken after 10:00 p.m. From a total of 101 cities of the State, 10 were randomly selected, Bancroftian filariasis was only found in Maceió, the capital of Alagoas State. In a cross-sectional survey conducted among the general population of 4 neighborhoods in the city, 10,973 individuals were examined, 226 were microfilaraemic with prevalence in the city neighborhoods ranging from 0 to $5.4 \%$. Prevalence rates and microfilariae density were significantly higher in males. Among the examined inhabitants born out of Maceió, microfilariae carriers had lived a significantly longer time in endemic areas than the amicrofilaraemic subjects. Based on the data obtained in the present study, measures to avoid expansion of bancroftian filariasis in the region were established.

Key-words: Wuchereria bancrofti. Lymphatic filariasis. Epidemiology.

\footnotetext{
1. Departamento de Patologia do Centro de Ciências Biológicas da Universidade Federal de Alagoas, Maceió, AL;2. Centro de Pesquisas Aggeu Magalhães/FIOCRUZ, Recife, PE; 3. Departamento de Parasitologia do Instituto de Ciências Biológicas da Universidade Federal de Minas Gerais, Belo Horizonte, MG.

Apoio financeiro: CNPq, Fundação Nacional de Saúde/AL e Secretaria Municipal de Saúde de Maceió.

Endereço para correspondência: Drª Eliana M. Mauricio da Rocha. Depto ${ }^{\circ}$ de Patologia/CCBi/UFAL. Praça Afrânio Jorge s/n, Prado 57010-020 Maceió, AL, Brasil.

Tel: 5582 327-3336; fax: 5582 221-2501.

e-mail: emmr@fapeal.br

Recebido para publicação em 5/4/99.
} 
A filariose linfática, também conhecida como elefantíase na sua fase crônica e sintomática, ocorre no Brasil exclusivamente pelo parasitismo de helmintos da espécie Wuchereria bancrofti. A bancroftose é endêmica em várias regiões tropicais, sendo estimado em 106 milhões o número de infectados no mundo ${ }^{20}$. No Brasil, entre 1951 a 1958, foram realizados vários inquéritos hemoscópicos e entomológicos, ficando constatada a transmissão autóctone da bancroftose em onze localidades, relacionadas a seguir com as respectivas prevalências: Ponta Grossa (SC), 13,9\%; Belém (PA), 9,8\%; Barra de Laguna (SC), 9,4\%; Recife (PE), 6,9\%; Castro Alves (BA), 5,9\%; Florianópolis (SC), 1,4\%; São Luis (MA), 0,6\%; Salvador (BA), 0,4\%; Maceió (AL), $0,3 \%$; Manaus (AM), $0,2 \%$ e Porto Alegre (RS), $0,1 \%{ }^{14}$. O tratamento das fontes de infecção, naquela época, levou a uma apreciável redução nas taxas de prevalência, sendo quase todos os focos considerados extintos, exceto, segundo o Ministério da Saúde, aqueles existentes em Belém (PA) e Recife (PE) ${ }^{10}$. No entanto, o encontro de três portadores autóctones de $W$. bancrofti em Maceió-AL em 19905, mostrou a necessidade de uma reavaliação da parasitose na área, que foi feita através de amostragem de estudantes de escolas noturnas, oriundos de todos os 33 bairros da cidade 6 . O estudo mostrou uma distribuição focal da parasitose, já que $85 \%$ dos parasitados encontrados eram residentes em apenas três bairros centrais e contíguos, com prevalências variando de $1,2 \%$ a $5,3 \%{ }^{6}$.

O objetivo da presente investigação é verificar a distribuição da bancroftose na população geral (todas as faixas etárias) em Maceió e em cidades localizadas nas diferentes regiões fisiográficas do estado de Alagoas.

\section{MATERIAL E MÉTODOS}

Áreas estudadas. Estado de Alagoas: localizado na região centro-oriental do Nordeste do Brasil, com área de $29.106 \mathrm{Km}^{2}$ e população de 2.514.100 habitantes (1.482.033 na área urbana $)^{7}$, possui 101 municípios, divididos em três regiões fisiográficas: leste (litoral e zona da mata), agreste e sertão. Foram selecionados, por sorteio, $10 \%$ dos municípios de cada região, sendo cinco do leste (Coruripe, Marechal Deodoro, Paripueira, Porto Calvo e Maragogi), dois do agreste (Palmeira dos Índios e Maribondo) e dois do sertão (Piranhas e Pão de Açúcar). Maceió: (924'-935'S latitude e 3544'-3556'W longitude), capital de Alagoas, está situada no litoral médio do estado, com uma população urbana de 555.421 pessoas vivendo em área de $208 \mathrm{~km}^{2}$ subdividida em 33 bairros.

População estudada. As amostras de população geral foram obtidas em agrupamentos (cluster) e o consentimento verbal foi obtido de cada participante do estudo ou seu responsável, após compromisso de tratamento dos microfilarêmicos detectados. Postos de colheita de sangue foram instalados em diferentes locais nos bairros analisados, de maneira a cobrir toda a região a ser pesquisada. Momentos antes da colheita de sangue, eram ministradas palestras a fim de conscientizar os participantes do estudo sobre a biologia do parasito e vetores, modos de transmissão e medidas de controle da parasitose. Em Maceió, amostras foram obtidas entre voluntários dos três bairros de maior prevalência para a bancroftose (Feitosa, Pitanguinha e Jacintinho) e de um bairro onde não foi encontrada a parasitose (Chã da Jaqueira), segundo estudos prévios com escolares ${ }^{6}$. Os três bairros de maior endemicidade amostrados são adjacentes e se encontram de três a cinco km distantes do bairro amostrado onde não foi detectada a parasitose entre escolares. As condições ambientais e sócioeconômicas são semelhantes nestes quatro bairros. $O$ cálculo do tamanho de amostra utilizou como estimativa as prevalências previamente encontradas entre escolares ${ }^{6}$, com erro máximo tolerável de $20 \%$ e intervalo de confiança de $95 \%$.

Nas áreas urbanas dos municípios selecionados do interior e litoral de Alagoas, foram obtidas amostras de voluntários de todas as faixas etárias, utilizando metodologia semelhante àquela aplicada em Maceió. Durante as colheitas de sangue foram levantados dados como: idade, sexo, naturalidade, tempo de residência na área e endereço das pessoas examinadas.

Pesquisa de microfilárias sanguíneas. 0 sangue era obtido por punção capilar digital e gota espessa qualitativa $(60-100 \mu \mathrm{l})$ era preparada sem uso de anticoagulantes para evitar a perda de microfilárias $(\mathrm{mf})^{11}$. A colheita era realizada entre 22:00h e 1:00h devido a periodicidade noturna das mf no sangue periférico do hospedeiro na região ${ }^{16}$. Após desemoglobinizadas, as gotas espessas eram coradas em eosina-Giemsa. Só foram considerados autóctones os microfilarêmicos que nasceram na área em estudo e/ou nunca 
viveram em outras regiões endêmicas para a bancroftose.

Densidade de microfilárias (mf). Dos parasitados foram colhidos, em torno das 23:00h, $3 \mathrm{ml}$ de sangue através de punção venosa para quantificação de mf pela técnica de filtração em membrana de policarbonato (Nuclepore Corporation, Pleasanton, CA, USA). Indivíduos com microfilaremia menor ou igual a $100 \mathrm{mf} / \mathrm{ml}$ de sangue foram considerados de baixa parasitemia; entre 101 e $500 \mathrm{mf} / \mathrm{ml}$ de sangue foram considerados de média parasitemia e, com mais que $500 \mathrm{mf} / \mathrm{ml}$ de sangue foram considerados de alta parasitemia ${ }^{19}$.

Análise dos dados. Através do pacote estatístico EPIINFO version $6.02^{3}$. O $\chi^{2}$ e teste " $t$ " de Student foram usados para comparar proporções e médias, respectivamente; riscos foram estimados por odds relativa $(\mathrm{OR})^{8}$ ou razão de chance.

\section{RESULTADOS}

Prevalência de microfilarêmicos na população geral de Maceió. Nos quatro bairros estudados, foram examinados 10.973 indivíduos (Feitosa 2.450, Pitanguinha 1.865, Jacintinho 4.637 e Chã da Jaqueira 2.021) de todas as faixas etárias, entre agosto de 1993 e abril de 1996, compreendendo $9,4 \%$ da população (variando de $6,7 \%$ a $24,2 \%$ ).
A Tabela 1 apresenta as prevalências de microfilarêmicos nos respectivos bairros. Considerando os três bairros endêmicos e contíguos, a prevalência média foi de $2,5 \%$.

A idade dos examinados variou de 2 a 86 anos e entre os microfilarêmicos de 5 a 57 anos, não sendo as médias de idade entre microfilarêmicos

Tabela 1 - Número de moradores, indivíduos examinados na população geral e microfilarêmicos por Wuchereria bancrofti em diferentes bairros de Maceió, $A L$.

\begin{tabular}{|c|c|c|c|c|c|}
\hline \multirow[b]{2}{*}{ Bairro } & \multirow[t]{2}{*}{ População* } & \multicolumn{2}{|c|}{ Examinados } & \multicolumn{2}{|c|}{ Positivos } \\
\hline & & $\mathrm{n}^{0}$ & $\%$ & $\mathrm{n}^{0}$ & $\%$ \\
\hline Feitosa & 17.599 & 2.450 & 13,9 & 133 & 5,4 \\
\hline Pitanguinha & 7.701 & 1.865 & 24,2 & 43 & 2,3 \\
\hline Jacintinho & 69.567 & 4.637 & 6,7 & 50 & 1,1 \\
\hline Chã da Jaqueira & 22.045 & 2.021 & 9,2 & 0 & 0,0 \\
\hline
\end{tabular}

${ }^{*}$ Fonte: IBGE${ }^{\text {. }}$.

(21,2 $\pm 9,9$ anos) e amicrofilarêmicos $(23,1 \pm 14,1$ anos) estatisticamente diferentes $(p>0,05)$.

Dos 226 microfilarêmicos detectados, 199 $(88,1 \%)$ são nascidos e criados em Maceió, 22 $(9,7 \%)$ provenientes do interior de Alagoas e 5 $(2,2 \%)$ provenientes de outros Estados (dois de Pernambuco, um de Sergipe, um de São Paulo, um do Distrito Federal). Entre os migrantes, o tempo médio de residência em Maceió foi significativamente maior entre os 27 microfilarêmicos (12,8 $\pm 5,9$ anos) que entre os não infectados examinados $(7,8 \pm$ 5,1 anos) $(p<0,05)$. Todos os microfilarêmicos foram considerados autóctones de Maceió; os nascidos em Maceió, viveram o tempo todo nos bairros estudados e os parasitados não nascidos em Maceió vieram de áreas indenes e moravam nos bairros endêmicos em média há mais de 10 anos.

Com relação ao sexo, dos 10.973 examinados, $4.690(42,7 \%)$ eram masculinos e $6.283(57,3 \%)$ eram femininos, proporção muito semelhante à distribuição por sexo em Maceió, onde 46,8\% da população é masculina e $53,2 \%$ é feminina ${ }^{7}$. A Tabela 2 apresenta a distribuição de microfilarêmicos em relação ao sexo. A odds relativa de estar parasitado entre masculinos quando comparados com femininos é 3,02 (I.C. 95\%: 2,26-4,05). A prevalência de microfilarêmicos é maior na população jovem, de ambos os sexos, aumentando com a idade e atingindo um platô em torno de 21-25 anos nos homens e entre 16-20 anos nas mulheres. A partir daí decresce com o avanço da idade (Figura 1).

Prevalência de microfilarêmicos em áreas urbanas no interior e litoral de Alagoas. A amostra total de 20.103 voluntários incluiu cerca de $25 \%$ das populações urbanas de 8 municípios selecionados (variando de 18,9\% a 45,6\%), exceção para Palmeira dos Índios onde, devido a dificuldades operacionais e tamanho da população, foram examinados 2.149 voluntários (4,8\% da população). 
Tabela 2 - Distribuição de microfilarêmicos por Wuchereria bancrofti de acordo com o sexo na população geral dos três bairros mais endêmicos para bancroftose em Maceió, $A L$.

\begin{tabular}{|c|c|c|c|c|c|c|}
\hline \multirow[b]{2}{*}{ Bairro } & \multirow[b]{2}{*}{ Sexo } & \multicolumn{2}{|c|}{ Microfilarêmicos } & \multicolumn{2}{|c|}{ Não microfilarêmicos } & \multirow[b]{2}{*}{ Odds relativa (IC 95\%) } \\
\hline & & $\mathrm{n}^{0}$ & $\%$ & $\mathrm{n}^{0}$ & $\%$ & \\
\hline \multirow[t]{2}{*}{ Feitosa } & M & 88 & 8,2 & 991 & 91,8 & $2,62(1,78-3,86)$ \\
\hline & $\mathrm{F}$ & 45 & 3,3 & 1.326 & 96,7 & \\
\hline \multirow[t]{2}{*}{ Pitanguinha } & M & 33 & 4,5 & 707 & 95,5 & $5,20(2,43-11,42)$ \\
\hline & $\mathrm{F}$ & 10 & 0,9 & 1.115 & 99,1 & \\
\hline \multirow[t]{2}{*}{ Jacintinho } & M & 33 & 1,7 & 1.917 & 98,3 & $2,70(1,44-5,10)$ \\
\hline & $\mathrm{F}$ & 17 & 0,6 & 2.670 & 99,4 & \\
\hline \multirow[t]{2}{*}{ Total } & M & 154 & 4,1 & 3.615 & 95,9 & $3,02(2,26-4,05)$ \\
\hline & $\mathrm{F}$ & 72 & 1,4 & 5.111 & 98,6 & \\
\hline
\end{tabular}

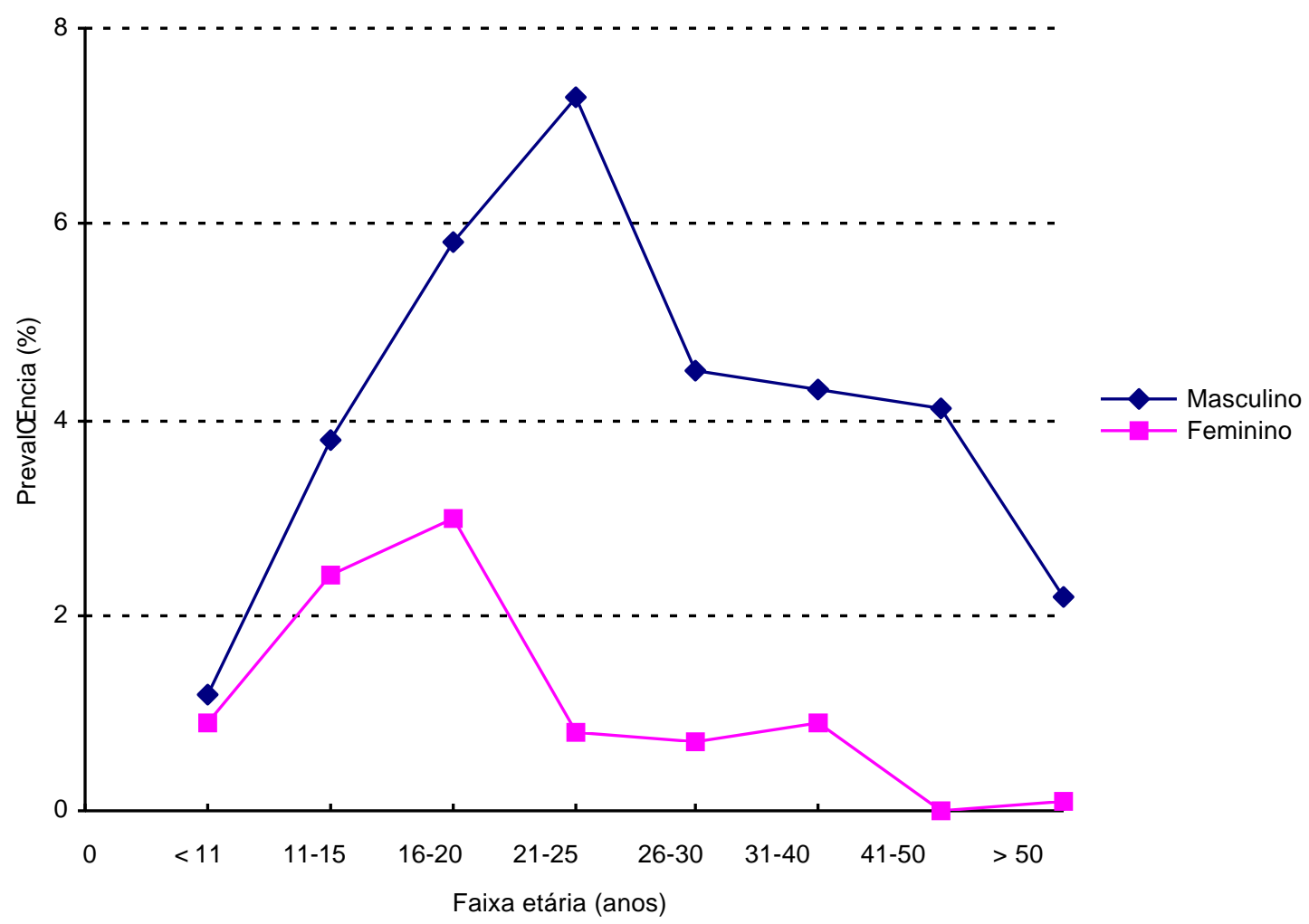

Figura 1 - Prevalência de microfilarêmicos por Wuchereria bancrofti, por sexo e faixa etária na população geral de Maceió, AL.

A Tabela 3 mostra as populações e amostras analisadas. Em 8 cidades pesquisadas não foi detectado nenhum microfilarêmico. Em Paripueira, município litorâneo situado a $25 \mathrm{~km}$ ao Norte de Maceió, foram detectados, entre 2.205 examinados, $2(0,09 \%)$ microfilarêmicos por $W$. bancrofti, sendo um do sexo masculino e outro feminino, com 8 e 19 anos de idade, respectivamente. Estes dois parasitados não foram considerados autóctones de Paripueira uma vez que residiam nesta cidade há menos de oito meses, tempo inferior ao período prépatente da parasitose e eram originários do bairro Feitosa de Maceió. 
Tabela 3 - População urbana e amostras examinadas para pesquisa de microfilarêmicos por Wuchereria bancrofti em municípios do interior e litoral de Alagoas.

\begin{tabular}{|c|c|c|c|c|c|c|}
\hline \multirow[t]{2}{*}{ Cidade } & \multirow[t]{2}{*}{ Região } & \multirow[t]{2}{*}{ População urbana* } & \multicolumn{2}{|c|}{ Examinados } & \multicolumn{2}{|c|}{ Positivos } \\
\hline & & & $\mathrm{n}^{\circ}$ & $\%$ & $\mathrm{n}^{\circ}$ & $\%$ \\
\hline Paripueira & Leste & 7.914 & 2.205 & 27,9 & 2 & 0,09 \\
\hline Maragogi & Leste & 6.667 & 1.795 & 26,5 & 0 & - \\
\hline Porto Calvo & Leste & 11.182 & 2.989 & 26,7 & 0 & - \\
\hline Marechal Deodoro & Leste & 14.658 & 2.765 & 18,9 & 0 & - \\
\hline Coruripe & Leste & 12.710 & 2.479 & 19,5 & 0 & - \\
\hline Palmeira Índios & Agreste & 44.596 & 2.149 & 4,8 & 0 & - \\
\hline Maribondo & Agreste & 9.632 & 1.975 & 20,5 & 0 & - \\
\hline Piranhas & Sertão & 1.718 & 789 & 45,6 & 0 & - \\
\hline Pão Açúcar & Sertão & 8.504 & 2.957 & 34,8 & 0 & - \\
\hline
\end{tabular}

*Fonte: IBGE?.

Densidade de $\mathrm{mf}$. A densidade absoluta $(\mathrm{mf} / \mathrm{ml}$ de sangue) entre os 226 parasitados variou de 1 a $4.130 \mathrm{mf} / \mathrm{ml}$, com média $580,5 \pm 786 \mathrm{mf} / \mathrm{ml}$. A densidade média de parasitemia entre indivíduos masculinos foi $706 \pm 868,8 \mathrm{mf} / \mathrm{ml}$ e entre os do sexo feminino foi $368,3 \pm 565,6 \mathrm{mf} / \mathrm{ml}$, sendo a diferença estatisticamente significativa $(p<0,05)$. Devido a grande variação nestas contagens, as parasitemias também foram analisadas através de médias geométricas. Assim, a média geométrica de $\mathrm{mf}$ entre os parasitados foi $2,25 \pm 0,87 \mathrm{mf} / \mathrm{ml}$. Entre os parasitados masculinos foi $2,35 \pm 0,89$ $\mathrm{mf} / \mathrm{ml}$ e entre os do sexo feminino, 2,08 $\pm 0,82 \mathrm{mf} /$ $\mathrm{ml}$, sendo a diferença estatisticamente significativa $(p<0,05)$. A Tabela 4 mostra a densidade de $\mathrm{mf}$ por sexo. Entre os parasitados masculinos, 42,9\% possuiam microfilaremia acima de $500 \mathrm{mf} / \mathrm{ml}$ (alta parasitemia) e entre os microfilarêmicos femininos apenas $16,7 \%$ eram portadores de alta parasitemia, sendo a diferença significativa $(p<0,01)$.

Tabela 4 - Distribuição de freqüência da microfilaremia por Wuchereria bancrofti de acordo com sexo, nas áreas mais endêmicas de Maceió, AL.

\begin{tabular}{|c|c|c|c|c|c|c|}
\hline \multirow{2}{*}{$\begin{array}{l}\text { Microfilaremia } \\
(\mathrm{mf} / \mathrm{ml})^{*}\end{array}$} & \multicolumn{2}{|c|}{ Masculinos } & \multicolumn{2}{|c|}{ Femininos } & \multicolumn{2}{|c|}{ Total } \\
\hline & no parasitados & $\%$ & $\mathrm{n} \cong$ parasitados & $\%$ & $\mathrm{n}^{\circ}$ parasitados & $\%$ \\
\hline $1-100$ & 41 & 26,6 & 28 & 38,9 & 69 & 30,5 \\
\hline $101-500$ & 47 & 30,5 & 32 & 44,4 & 79 & 35,0 \\
\hline $501-1000$ & 22 & 14,3 & 8 & 11,1 & 30 & 13,3 \\
\hline$>1000$ & 44 & 28,6 & 4 & 5,6 & 48 & 21,2 \\
\hline Total & 154 & 100,0 & 72 & 100,0 & 226 & 100,0 \\
\hline
\end{tabular}

${ }^{*} \mathrm{mf} / \mathrm{ml}=$ número de microfilárias $/ \mathrm{ml}$ de sangue.

\section{DISCUSSÃO}

Um processo formal de amostragem aleatória de participantes não foi possível de ser realizado por razões operacionais: seria necessário a construção de listas censitárias da população dos bairros e municípios incluídos na investigação, uma vez que as mesmas não estavam disponíveis. Optou-se pela amostragem em agrupamentos ou conglomerados (cluster), identificados a partir da inclusão de voluntários no estudo. O principal problema na utilização de amostras por conglomerado reside no fato das mesmas poderem apresentar uma variância maior quando comparadas a amostras aleatórias. Isto poderia impor sérias limitações à interpretação dos resultados obtidos, devido a heterogeneidade da amostra, resultando em imprecisão das estimativas obtidas. Entretanto, assumindo que 1) os conglomerados construídos na presente investigação podem ser considerados como agrupamentos aleatórios de indivíduos, residentes do mesmo bairro ou comunidade, a variância da amostra por conglomerado não é maior do que a variância de uma amostra aleatória, sendo vantajoso utilizar os benefícios práticos associados à amostragem por conglomerado; e 
2) mesmo que os conglomerados construídos no estudo apresentassem uma maior homogeneidade com relação a fatores de risco para infecção por $W$. bancrofti do que aquela que se esperaria por chance, o que resultaria em amostras com maior variância, as amostras utilizadas na investigação foram de tamanho suficiente para que as estimativas de riscos obtidos apresentassem o grau de precisão desejado ${ }^{8}$.

No passado, o único levantamento para detectar infecção por $W$. bancrofti na população geral de Maceió foi realizado por Deane et $\mathrm{al}^{4} \mathrm{em}$ 1953, sendo encontrado 0,3\% de microfilarêmicos. Naquela época já se verificava uma distribuição focal da parasitose uma vez que $67 \%$ dos parasitados foram detectados no bairro Farol. Recentemente foi verificado que entre estudantes de escolas noturnas da cidade, $85 \%$ dos parasitados eram procedentes dos bairros Feitosa, Jacintinho e Pitanguinha ${ }^{6}$, que são hoje contíguos ao bairro Farol, sugerindo que o foco atual seja uma extensão do foco original descrito há quase 50 anos. Considerando que em ambas as investigações as amostras fossem representativas das respectivas populações, pode-se afirmar que a proporção de parasitados na corrente investigação foi significativamente maior $(p<0,01)$ que a reportada em 1953 e este aumento pode estar associado ao acentuado crescimento da população urbana de Maceió e as precárias condições de vida da população no foco. Somente $27 \%$ da população de Maceió é servida por sistema de rede de esgotos ${ }^{7}$ e a carência em saneamento básico, com água estagnada e poluída no domicílio e peridomicílio, propicia condições adequadas à proliferação de criadouros do mosquito vetor, fator determinante na manutenção da filariose endêmica nas áreas urbanas.

A prevalência da bancroftose verificada na população geral não foi estatisticamente diferente daquela detectada previamente entre os escolares noturnos da mesma área ${ }^{6}$. Esses dados, aliado ao fato de que as maiores prevalências de bancroftose ocorrem em grupos etários mais jovens ${ }^{15}$, podem explicar porque a amostra de escolares noturnos examinada previamente ${ }^{6}$, com média de idade 18,4 \pm 4,6 anos, foi representativa para a distribuição de microfilarêmicos na população geral da área urbana da capital alagoana que possui uma média de idade 24,0 \pm 18,2 anos ${ }^{7}$. Assim, a amostra de escolares noturnos que é mais fácil de ser obtida, pode ser adequada para estudos futuros de avaliação de programas de controle da parasitose na área, diminuindo tempo e custos operacionais.

Os municípios amostrados no interior de Alagoas não apresentaram nenhum caso de bancroftose autóctone, embora a maior parte destas cidades sejam pobres, carentes em saneamento básico, com grande população vetorial e aglomerado de pessoas em pequenas áreas urbanas. Aparentemente, a parasitose ainda não se instalou fora da capital alagoana, apesar de municípios como Maragogi e Porto Calvo ficarem localizados entre Maceió e Recife, focos de transmissão ativa de bancroftose no país. Porém, com o crescente intercâmbio migratório, existe o risco da bancroftose ser reintroduzida ou introduzida em áreas consideradas indenes. Este tipo de situação já ocorreu em localidades no Sri Lanka, onde migrantes infectados originaram focos da parasitose em zonas onde antes não existia a filariose linfática ${ }^{18}$.

A distribuição da bancroftose por sexo e idade mostrou que a prevalência entre homens foi sempre maior que entre mulheres, sendo esta diferença significativa dos 16 aos 50 anos. Esses dados são semelhantes aos observados em Samoa ${ }^{9}$ mas, no entanto, em alguns locais como no Haiti, não foram detectadas diferenças com relação ao sexo ${ }^{12}$. No Brasil, foi mostrado recentemente, prevalências superiores entre indivíduos masculinos, sendo a diferença significativa dos 15 aos 54 anos $^{1}$. Apesar dos mecanismos que resultam numa maior resistência à infecção pelas mulheres não serem ainda conhecidos, estudos sugerem que os hormônios femininos podem influenciar a parasitemia em filariose bancroftiana ${ }^{13}$. Fatores hormonais associados com a gravidez podem restringir a fertilidade dos vermes adultos ou tornar o hospedeiro mais refratário à infecção². Existem fortes evidências que ocorra resistência imunológica à reinfecção com o aumento da idade mas, como nem todos que vivem em região endêmica tornam-se infectados, acredita-se que existe também uma imunidade natural às larvas infectantes ${ }^{17}$.

A microfilaremia média significativamente maior entre masculinos e, independente do sexo, $34,5 \%$ dos microfilarêmicos possuirem altas densidades de $\mathrm{mf}$ coincidem com os dados obtidos em Recife, onde 33,4\% dos portadores apresentavam microfilaremia maior que $500 \mathrm{mf} / \mathrm{ml}$ de sangue ${ }^{1}$. A determinação da microfilaremia 
assume importância em estudos de transmissibilidade da bancroftose porque permite, juntamente com dados relativos a densidade de vetores na área e sua capacidade de transmissão, o planejamento de programas de controle adequados para as características de cada região.

É objetivo da Organização Mundial de Saúde (OMS) e Organização Pan Americana de Saúde (OPAS) a eliminação da Filariose linfática das Américas, uma vez que a OMS inclui esta enfermidade entre as doenças potencialmente erradicáveis ${ }^{20}$. Baseado nos dados desta investigação, medidas de controle já foram implementadas, envolvendo o Ministério da Saúde e orgãos de Saúde Pública regionais, visando a eliminação da bancroftose na região. O sucesso deste programa evitará problemas futuros como o aparecimento de casos clínicos crônicos, que podem apresentar alterações que são algumas vezes irreversíveis.

\section{REFERÊNCIAS BIBLIOGRÁFICAS}

1. Albuquerque MF, Marzochi MC, Sabroza PC, Braga MC, Padilha T, Silva MC, Silva MR, Schindler HC, Maciel MA, Souza W, Furtado AF. Bancroftian filariasis in two urban areas of Recife, Brazil: pre-control observations on infection and disease. Transactions of the Royal Society of Tropical Medicine and Hygiene 89:373-377, 1995.

2. Brabin L. Sex differentials in susceptibility to lymphatic filariasis and implications for maternal child immunity. Epidemiology and Infections 105:335-353, 1990.

3. Dean AG, Dean JA, Coulombier D, Brendel KA, Smth DC, Burton AH, Dicker RC. Epi Info, version 6.02; a word processing, database, and statistics program for epidemiology on micro-computers. Atlanta: Center of Disease Control, 1994

4. Deane LM, Rosa D, Rachou RG, Martins JS, Costa A, Gomes HM, Carvalho ME. A filariose bancroftiana em Maceió, Alagoas: Resultado de um inquérito realizado em 1952. Revista Brasileira de Malariologia e Doenças Tropicais 5:17-22, 1953.

5. Dreyer G, Béliz F, Vergetti G, Vergetti A, Café T, Fontes G. Autochthonous Wuchereria bancrofti microfilaremia in the city of Maceió-Alagoas-Brazil. Memórias do Instituto Oswaldo Cruz 86:495-496, 1991.

6. Fontes G, Rocha EM, Brito AC, Antunes CMF. Lymphatic filariasis in Brazilian urban area (Maceió, Alagoas). Memórias do Instituto Oswaldo Cruz 93:705-710, 1998.

7 Instituto Brasileiro de Geografia e Estatística (IBGE) - X Recenseamento Geral Brasileiro, (Alagoas, Censo Demográfico, Dados Distritais), n.15, p.1-115, 1991.

8. Kahn HA, Sempos CT. Statistical Methods in Epidemiology. Monographs in Epidemiology and Biostatistics, Oxford University Press, New York, vol. 12, 1989.

9. McCarthy DD, Fitzgerald N. Habit, Habitat and Hyperfilariation in the epidemiology of filariasis in Western Samoa. Transactions of the Royal Society of Tropical Medicine and Hygiene 50:58-65, 1956.

10. Ministério da Saúde. O controle das Endemias no Brasil (de 1979 a 1984). Brasília (DF): Superintendência de Campanhas de Saúde Pública, SUCAM, 1985.

11. Partono F, Idris KN. Some factors influencing the loss of microfilariae from stained thick blood films. Southeast
Asian Journal of Tropical Medicine and Public Health 8:158-164, 1977

12. Raccurt CP, Lowrie Jr RC, Katz SP, Duverseau YT. Epidemiology of Wuchereria bancrofti in Leogane, Haiti. Transactions of the Royal Society of Tropical Medicine and Hygiene 82:721-725, 1988.

13. Raccurt CP, Mojon M, Hodges WH. Parasitological, serological and clinical studies of Wuchereria bancrofti in Limbe, Haiti. American Journal of Tropical Medicine and Hygiene 33:1124-1129, 1984.

14. Rachou RG. Conceito e programa de profilaxia da filariose bancroftiana no Brasil. Revista Brasileira de Malariologia e Doenças Tropicais 12:11-40, 1960.

15. Rajasekariah GR, Parab PB, Chandrashekar R, Deshpande L, Subrahmanyam D. Pattern of Wuchereria bancrofti microfilaraemia in young and adolescent school children in Bassein, India, an endemic area for lymphatic filariasis. Annals of Tropical Medicine and Parasitology 85:663-665, 1991.

16. Rocha EMM, Fontes G, Vergetti G, Santos ACB, Fireman FAT, Dreyer G. Periodicidade de microfilárias de Wuchereria bancrofti em filarióticos autóctones de Maceió-AL. Revista do Instituto de Medicina Tropical de São Paulo 33:35, 1991.

17. Vanamail P, Subramanian S, Das PK, Pani SP, Rajagopalan PK, Bundy DA, Grenfell BT. Estimation of age-especific rates of acquisition and loss of Wuchereria bancrofti infection. Transactions of the Royal Society of Tropical Medicine and Hygiene 83:689-693, 1989.

18. World Health Organization (WHO). Filariasis linfática: Cuarto informe del Comité de Expertos de la OMS en Filariasis. Ginebra. Serie de Informes Técnicos, n.702, 1984.

19. World Health Organization (WHO). Lymphatic filariasis: the disease and its control. Fifth report of the WHO Expert committee on filariasis. Geneva. Technical reports series, n.821, 1992.

20. World Health Organization (WHO). Lymphatic filariasis infection \& disease: control strategies. Report of a $\mathrm{WHO} /$ CTD/TDR consultative meeting held at the University Sains Malaysia, TDR/CTD/FIL/PENANG/94.1, 1994. 